\title{
A Plasma Klystron for Generating Ultra-Short Electron Bunches
}

\author{
Thomas C. Katsouleas, Fellow, IEEE, C. E. Clayton, L. Serafini, C. Pellegrini,
} C. Joshi, Fellow, IEEE, J. Dawson, and P. Castellano

\begin{abstract}
A technique for producing ultra-short electron bunches (e.g., $\leq \mathbf{1 0 0}$ fs) from a continuous electron beam using a short plasma wave section and a drift space is explored. The bunches are a fraction of a plasma wavelength long and are spaced by a plasma wavelength, making them of interest for injection into plasma accelerators or for driving a klystron-like structure to produce infrared radiation.
\end{abstract}

\section{INTRODUCTION}

$\mathbf{T}$ ECHNIQUES for producing ultra-short bunches (e.g., less than $100 \mathrm{fs}$ ) of electrons are of interest for a variety of applications including plasma accelerators, pulsed radiolysis (time-resolved ultra-fast chemistry), and high-power infrared light sources. With the success of recent proof-of-principle plasma accelerator experiments (see, for example, papers in this issue by Modena et al. and Amiranoff et al.), the need for synchronously injected bunches that are a fraction of a plasma wavelength (typically $300 \mu$ or less) is particularly acute to advance that field to the next milestone: production of high quality beams.

Previous work on ultra-short bunches has been focused largely on magnetic compression of longer beams that are given a correlated energy spread by an RF field. Kung et al. [1] used a thermionic cathode and an RF accelerating structure to produce a bunch of $2 \mathrm{MeV}$ electrons with a linear energy ramp from back to front. An alpha magnet was then used to compress the bunch to $50 \mathrm{fs}$. Carlsten et al. [2] used an RF photocathode and magnetic chicane to compress a $1 \mathrm{nC}$ bunch at $8 \mathrm{MeV}$ to less than $1 \mathrm{ps}$.

In this paper we explore an alternative concept to producing short bunches using a short plasma wave section and drift space.

This has several advantages for plasma accelerators including simplicity and absolute synchronism of the bunches with the accelerating plasma wave. Moreover, the technique provides well-bunched multiple beams that could be used in a klystron-like structure. This might produce high-power infrared radiation in a wavelength regime $(\lambda \sim 100 \mu \mathrm{m})$ that is now difficult to access with inexpensive devices.

Manuscript received January 30, 1996; revised February 9, 1996. This work was supported in part by the U.S. Department of Energy under Grant DEFG03-92ER40745.

T. C. Katsouleas and P. Castellano are with the University of Southern California, Los Angeles, CA 90089-0484 USA (e-mail: katsoule@usc.edu).

C. E. Clayton, C. Pellegrini, C. Joshi, and J. Dawson are with the University of California, Los Angeles, CA 90024 USA.

L. Serafini is with the INFN-Milan, 20133 Milano, Italy.

Publisher Item Identifier S 0093-3813(96)03861-1.

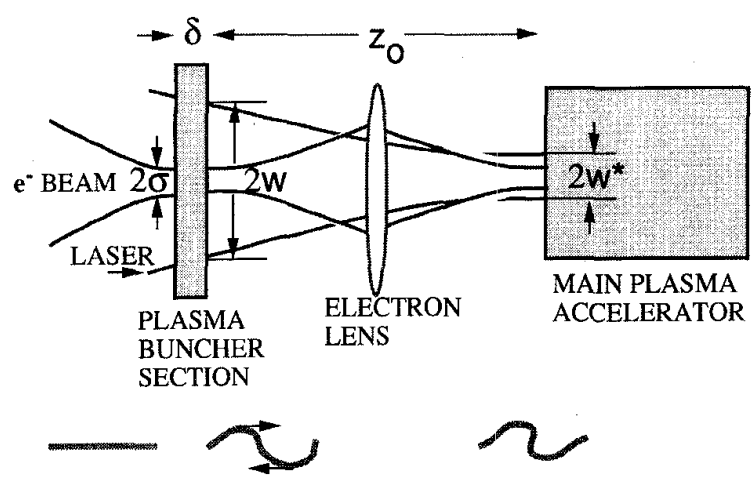

Fig. 1. Schematic of plasma buncher. By moving the buncher section slightly (changing the drift length $z_{0}$ ), the phase of the bunches injected into a plasma accelerator can be controlled. A cartoon of longitudinal phase space snapshots of the beam at three positions is shown below.

The basic idea of the plasma buncher we propose is illustrated in Fig. 1. A thin plasma section of length $\delta$ is driven by a driver laser (laser wakefield or beat wave excitation [3]). The long electron bunch receives a longitudinal momentum kick from the plasma wave causing it to bunch a distance $z_{0}$ downstream. This yields periodic bunches separated by the plasma wavelength, $\lambda=2 \pi c / \omega_{p}\left(\omega_{p}^{2} \equiv 4 \pi n_{0} e^{2} / m, n_{0}\right.$ is plasma density).

From the figure several advantages of this scheme are apparent. First, as a buncher for a laser-driven plasma accelerator the scheme only requires the insertion of a thin plasma slab (e.g., a gas jet) into the beam-line of an unbunched plasma accelerator. No additional RF or magnetic chicanes are needed (an electron focusing element is required and is discussed later). Second, it is easy to see how phasing of the electrons in the plasma accelerator can be accomplished by moving the plasma slab (i.e., varying the drift length $z_{0}$ ) slightly. Since the electrons move at velocity $v_{b}<c$, they slip behind the laser by a distance of $z_{0} / 2 \gamma^{2}$ ( $\gamma$ is the electrons' Lorentz factor) during the drift space. Thus, small changes in $z_{0}$ lead to very fine tuning of the phase of the injected electrons with respect to the laser and hence the plasma accelerating wave.

The organization of the remainder of this paper is as follows. First, we summarize the design equations for the buncher section position $\left(z_{0}\right)$ and thickness $(\delta)$. Then we consider the constraints on the design imposed by transverse effects and energy spread that cause bunch lengthening. An example is given for a current beat wave accelerator experiment at UCLA and the predictions are compared to a test particle 
simulation model. We also consider the use of harmonic or nonlinear plasma wave steepening to improve the bunching effectiveness. Finally, estimates of the minimum bunch length that can be obtained for both emittance-dominated and spacecharge dominated beams are presented.

\section{SCALING LAWS}

The position of the buncher is given by the drift length $z_{0}=$ $\gamma^{2} \Delta z\left(\frac{\gamma}{\Delta \gamma}\right)$, where $\Delta z / c$ is the difference in time-of-flight and $\Delta \gamma$ is the difference in energy imparted to electrons at the maximum and minimum accelerating field in the buncher. Thus $\Delta \gamma=2 e E_{0} \delta / m c^{2}$, where $\delta$ is the buncher thickness and $E_{0}$ is the plasma wave amplitude in the buncher. We wish to choose $\Delta z=\lambda_{p} / 2$ to obtain maximum bunching where $\lambda_{p}=2 \pi c / \omega_{p} \approx 300 \mu \times\left(10^{17} \mathrm{~cm}^{-3} / n_{0}\right)^{1 / 2}$. We have left to determine $E_{0}$. For short pulses the plasma wave amplitude will be proportional to laser intensity which decreases as the buncher is moved farther from the laser focus (i.e., as $z_{0}$ is made larger). Thus $E_{0} \approx E_{0}^{*} \frac{w^{* 2}}{w^{2}}$ where $E_{0}^{*}$ is the plasma wave amplitude at the laser focus, $w^{*}$ the laser spot size at its focus, and $w$ its size at the buncher. Finally, the laser waist spreads according to $w \approx z \theta \approx z_{0} \frac{k_{\perp}}{k_{\|}} \approx z_{0} \frac{2 / w^{*}}{2 \pi / \lambda_{0}}$, where $\lambda_{0}$ is the laser wavelength. Combining the above expressions gives an expression for the buncher thickness $(\delta)$ and position $(z)$ in terms of the electron beam, laser, and plasma accelerating field parameters

$$
\delta=\frac{\gamma^{3} \lambda_{0}^{2}\left(c^{2} / \omega_{p}^{2}\right) z_{0}}{2 \pi w^{* 4} \varepsilon^{*}}
$$

where $\varepsilon^{*}=e E_{0}^{*} / m c \omega_{p}$. Of course $\varepsilon^{*}$ depends on the laser power and spot size, but we have chosen to express $\delta$ in this way because $\varepsilon^{*}$ is typically designed to be in the range of $0.1-0.5$. For example, if $\gamma=20, \lambda_{0}=10 \mu$ (e.g., a $\mathrm{CO}_{2}$ laser), $w^{*}=500 \mu, \varepsilon^{*}=0.5, n_{0}=10^{16} \mathrm{~cm}^{-3}$, then $\delta=$ $0.0085 z_{0}$. Thus for a typical gas jet of $\delta=2.5 \mathrm{~mm}$ width, $z_{0}$ would be $30 \mathrm{~cm}$. By varying $z_{0}$ by $\pm 2 \mathrm{~cm}$, the position of the electron bunch can be moved $\pm 25 \mu \mathrm{m}$ with respect to the peak plasma wave accelerating field (approximately $\pm 30^{\circ}$ of phase).

A caveat must be made on laser power. The above scenario assumes that the laser is intense enough at the buncher to tunnel ionize the gas jet. This requires $I \geq 10^{14} \mathrm{~W} / \mathrm{cm}^{2}$ at the jet, corresponding to $I^{*}=\left(w^{2} / w^{* 2}\right) 10^{14} \mathrm{~W} / \mathrm{cm}^{2}\left(=10^{16}\right.$ $\mathrm{W} / \mathrm{cm}^{2}$ for our example) at the main plasma. This is typically not a problem for current high brightness $1 \mu$ lasers. On the other hand, the current $\mathrm{CO}_{2}$ laser at the UCLA Neptune Laboratory is about a factor of two too weak. In this case, it may be necessary to drive the buncher (gas jet) with a different laser from that driving the main plasma [4]. Although this scenario requires the additional step of synchronization of the two sets of lasers, it allows considerably more flexibility in the choice of beam, laser, and gas jet parameters. For this case, we re-express the scaling law above explicitly in terms of the plasma wave amplitude at the buncher $\left(\varepsilon_{0} \equiv e E_{0} / m c \omega_{p}\right)$

$$
\delta=\frac{\gamma^{3} \pi\left(c^{2} / \omega_{p}^{2}\right)}{2 \varepsilon_{0} z_{0}}
$$

\section{Constraints}

Next we consider several factors that can inhibit the ideal one-dimensional bunching scenario just described. First, it is clear that the energy spread in the beam $\left(\Delta \gamma_{b}\right)$ must be much less than the correlated energy spread $(\Delta \gamma)$ that the electrons receive from the buncher. Thus $\Delta \gamma_{b} / \gamma \ll \gamma^{2} \Delta z / z_{0}=$ $\pi \gamma^{2} c / \omega_{p} z_{0}$. For the example above, this gives $\Delta \gamma_{b} / \gamma \ll 0.1$.

Transverse effects can also limit the bunching. These include the effect of the electron beam emittance, the transverse variation of the plasma field in the buncher, and radial forces on the beam in the buncher. The electron beam's emittance causes the beam to spread radially in the drift section. If a thin focusing lens is used at the midpoint of the drift section, then it is easy to estimate the bunch lengthening caused by the added path length $(\Delta L)$ for electrons that are not on the axis: $\Delta L \approx z_{0}(1-\cos \theta) \approx z_{0} \theta^{2} / 2$, where $\theta$ is the angular spread of the electrons. To avoid bunch lengthening this requires

$$
\Delta L=z_{0} \theta^{2} / 2 \ll \Delta z=\pi c / \omega_{p}
$$

or

$$
\theta=\varepsilon / \pi \sigma \ll \sqrt{\frac{\pi c / \omega_{p}}{z_{0}}}
$$

For the previous example, this requires $\theta \ll 20 \mathrm{mrad}$. For a given emittance $(\varepsilon)$, this determines a minimum allowable spot size $(\sigma=\varepsilon / \pi \theta)$. That is, the beam cannot be too tightly focused at the buncher.

The nonzero spot size of the beam implies that an energy spread on the beam will result from the difference in the plasma wave amplitude on and off the symmetry axis. For a plasma wave proportional to the laser intensity and a Gaussian laser profile, the plasma wave amplitude as a function of $r$ will be $E(r)=E_{0} e^{-2 r^{2} / w^{2}} \approx E_{0}\left(1-2 r^{2} / w^{2}\right)$, where we assume $r \leq \sigma \ll w$. Thus we require $2 \sigma^{2} / w^{2} \ll \Delta \gamma / \gamma$ or

$$
\sigma \ll \frac{\omega}{\sqrt{2}} \sqrt{\frac{\Delta \gamma}{\gamma}} \approx \frac{\gamma w}{2} \sqrt{\frac{\pi c / \omega_{p}}{z_{0}}} \approx \frac{\gamma \lambda_{0}}{2 \pi w^{*}} \sqrt{\pi z_{0} / \omega_{p}}
$$

This gives a result that we have assumed earlier; namely, that at the buncher the electron beam should be much narrower than the laser. Evaluating this expression again for our example-gives $\sigma \ll 100 \mu$. The previous two inequalities imply a restriction on beam emittance. Combining these yields

$$
\varepsilon_{N} \equiv \gamma \varepsilon \ll \pi \frac{\gamma^{2} \lambda_{0} c / \omega_{p}}{2 w^{*}} .
$$

For our example, this gives $\varepsilon_{N} \ll 80 \pi \mathrm{mm}$-mrad, a very modest requirement.

The plasma wave in the buncher exerts radial as well as longitudinal forces on the beam. The maximum amplitude of this focusing/defocusing force is $F_{r}=4 r e E_{0} / k_{p} w^{2}$ [5]. Evaluating the angular kick $F_{r} \delta / \gamma m c$ at $r=\sigma$ and requiring that it be much less than the maximum angle given in (3) gives $\sigma \ll \frac{z_{0}^{3 / 2} \lambda_{0}^{2}}{8 \pi^{2} \sqrt{\pi} w^{* 2}\left(c / \omega_{p}\right)^{3 / 2}}$. This is typically a much softer requirement than (4); for our parameters it is $\sigma \ll 5 \mathrm{~cm}$. 


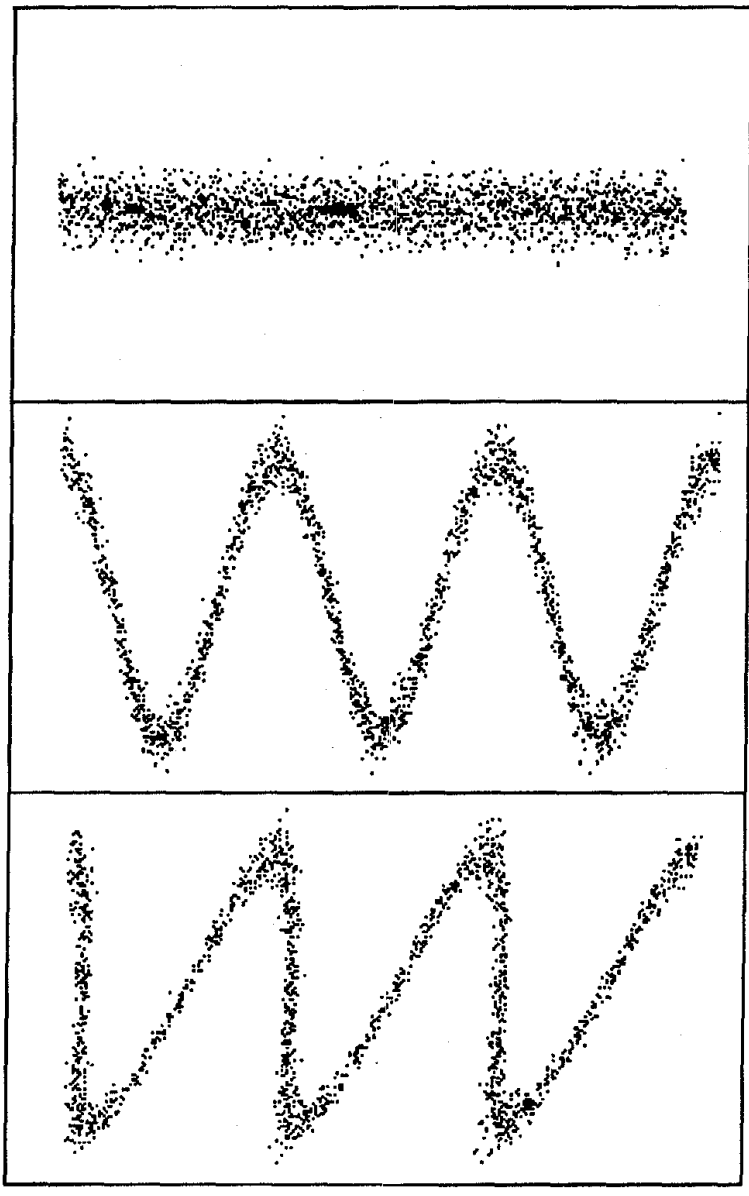

Fig. 2. Phase space, $p$ versus $z$, of the beam just before, just after the buncher, and at $z=z_{0}$ (2).

\section{Test Particle Simulations}

The simple scaling laws described above are now illustrated by numerical calculation of the evolution of a beam of 3000 particles, initially uniformly distributed in $z$ over three plasma wavelengths. The beam is Gaussian in $r$ with $\sigma=100 \mu, \gamma=$ 20 , energy spread of $0.1 \%$, and normalized emittance $\varepsilon_{N}=1 \pi$ $\mathrm{mm}$-mrad. The plasma wave, buncher, and laser parameters are

$$
\begin{aligned}
\text { plasma wave amplitude } \varepsilon_{0} & =0.038, \\
\text { plasma density } n_{0} & =10^{16} \mathrm{~cm}^{-3}, \\
\text { plasma wavelength } & =300 \mu, \\
\text { length of buncher } \delta & =2.5 \mathrm{~mm}, \\
\text { laser width } w & =1.5 \mathrm{~mm}, \\
\text { drift length } z_{0} & =30 \mathrm{~cm} .
\end{aligned}
$$

In Fig. 2 snapshots of the beam in phase space and real space are shown at $z=0, z=3 \mathrm{~mm}$ (just after the plasma slab), and $z=z_{0}$. In Fig. 3 , the bunching is quantified by calculating three relevant quantities: 1) the peak density enhancement of the bunched beam; 2) $\lambda / \mathrm{FWHM}$, the full width at half maximum of the bunched beam normalized to a plasma wavelength; and 3) the bunching parameter defined in FEL literature as $B=\frac{1}{N} \sum_{n=1}^{N} e^{i \phi_{n}}$, where $\phi_{n}=k_{p}(z-c t)_{n}$ is
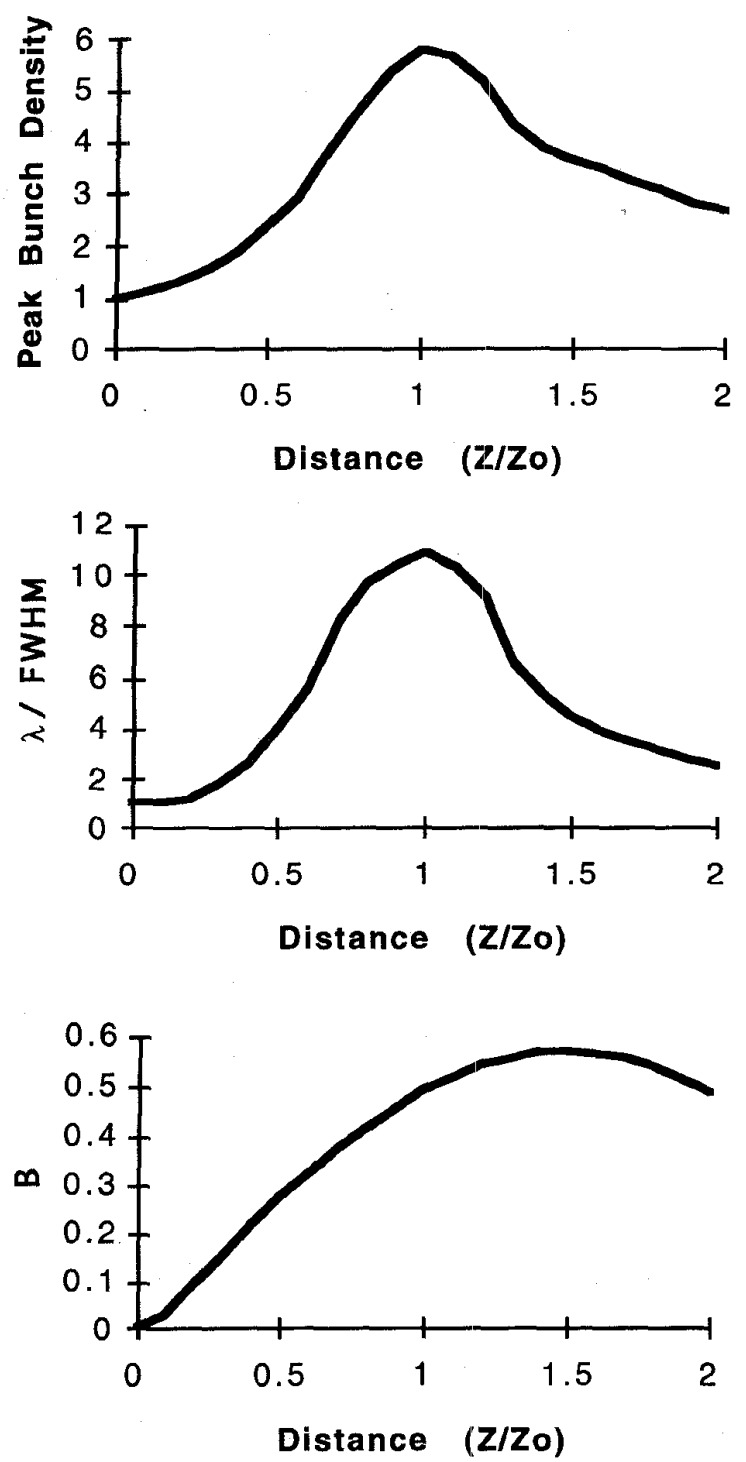

Fig. 3. Bunching as a function of drift distance from the plasma buncher [normalized to $z_{0}$ from (2)]: (a) peak electron bunch density (normalized to initial density of the continuous beam); (b) $(\mathrm{FWHM} / \lambda)^{-1}$; (c) bunching parameter $B$.

the phase of the $n$th particle. Fig. 3 shows that there is a broad peak in $\mathrm{FWHM}^{-1}$ and peak density at $1.5 z_{0}$, while $B$ peaks further away at $1.5 z_{0}$.

Ideally, a linear momentum chirp rather than a sinusoidal momentum kick would provide optimal bunching of the beam (in the absence of space charge [6]). For our plasma buncher this corresponds to a sawtooth plasma wave. Such waves arise naturally at very large amplitudes $(\varepsilon \approx 1)$ near wavebreaking. Such large amplitudes require very intense lasers and have longer (amplitude-dependent) wavelengths. Alternatively, steepend waves may be created at modest amplitudes by driving the plasma at its fundamental plus harmonics with appropriate amplitudes. This could be done by beating several rather than two laser lines simultaneously. To quantify the improvement in bunching afforded by this possibility, we show 


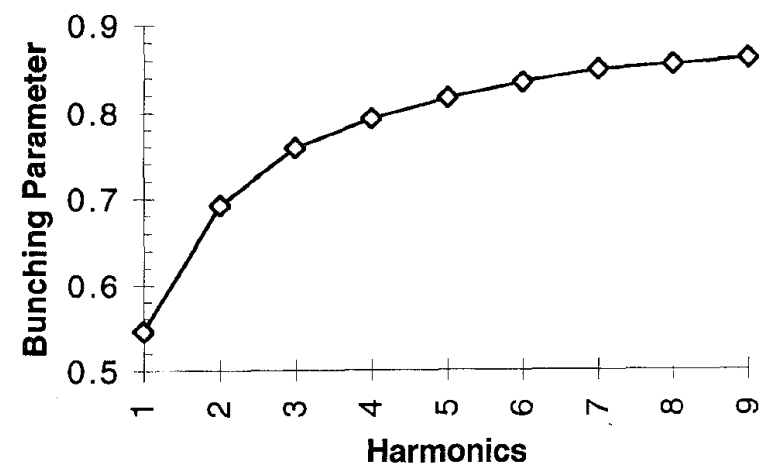

Fig. 4. Bunching parameter $B$ as a function of number of harmonics in the plasma wave (at $z=1.5 z_{0}$ ).

the results of numerical calculations of bunching parameter $B$ versus number of harmonics used in Fig. 4. That is, the plasma wave field is taken to be $E_{0} \sum_{n=1}^{N} \frac{(-1)^{n}}{n} \sin (n \psi)$, $\psi=k_{p} z-\omega_{p} t$. Note that a perfect sawtooth corresponds to an infinite number of harmonics.

\section{SPACE CHARGE}

The preceding arguments have described single particle effects. For very dense beams, the collective space charge of the beam can inhibit the bunching (i.e., the bunch length can become space charge rather than emittance dominated). We wish to evaluate analytically the debunching effect produced by the longitudinal space charge field in the drift region between the plasma slab and the beam-focus. We take into account that the beam is focusing both radially and longitudinally during the drift.

To derive a simple estimate of the importance of space charge, we will assume a single bunch model, with a charge density distribution which is uniform inside a cylinder of radius $R$ and length $L$. The evolution of these two quantities is a priori specified by assuming $R=R^{*} \sqrt{1+\left(z^{2} / \beta^{2}\right)}$, where $R^{*}$ is the focal spot size (at $z=0$ ) and $\beta=\frac{\gamma R^{-2}}{\varepsilon_{n}}$ while $L=L_{0}-\left(L_{0}-L^{*}\right)\left(z+z^{\prime}\right) / z^{\prime}$, and $z^{\prime}$ is the distance from the last lens to the focal point (i.e., $z^{\prime} \equiv z_{0} / 2$ ). The bunch starts therefore at $z=-z^{\prime}$ with transverse and longitudinal sizes $R_{0}=R^{*} \sqrt{1+\left(z_{0}^{2} / \beta^{2}\right)}$ and $L_{0}$. Assuming that the bunch aspect ratio $A_{r}$ in its rest frame (i.e., $A_{r}=\frac{R}{L-\gamma}$ ) is smaller than one during the entire drift (from $z=-z^{\prime}$ to $z=0$ ), the longitudinal space charge field $E_{z}$ at the bunch tail is given by $E_{z}=\frac{Q}{2 \pi \varepsilon_{0} R L \gamma}$, where $Q$ is the bunch charge. The bunch lengthening $\Delta L$, under the assumption of perturbative lengthening (i.e., $\Delta L \ll L^{*}$ ), will be given by

$$
\Delta L=\frac{2 e}{m c^{2} \gamma^{3}} \int_{z^{\prime \prime}}^{0} d z^{\prime \prime} \int_{-z^{\prime}}^{z^{\prime \prime}} E_{z} d z
$$

which, in turns, gives

$$
\Delta L=\frac{4 Q c}{I_{a}} \frac{\beta^{2}}{R^{*} L^{*} \gamma^{4}} f\left(a, x_{0}\right)
$$

where $I_{a}=17 \mathrm{kA}$, and the function $f\left(a, x_{0}\right)$ is given by

$$
\begin{aligned}
f\left(a, x_{0}\right)= & \left(1+a \cdot x_{0}\right) \\
& \times \frac{\left(\operatorname{ArcSinh}[a]-\log \left[\frac{\left(a-x_{0}+\sqrt{\left.\left(1+x_{0}^{2}\right)\left(1+a^{2}\right)\right)}\right.}{\left(1+a \cdot x_{0}\right)}\right]\right)}{\left(a \sqrt{\left.1+a^{2}\right)}\right.} \\
& -\frac{\operatorname{ArcSinh}\left[x_{0}\right]}{a}
\end{aligned}
$$

where $x_{0}=\frac{z_{0}}{2 \beta}$ and $a=\frac{\left(L_{0} / L^{*}-1\right)}{x_{0}}$.

In the case that $x_{0} \gg 1$ (as is typical) the function $f\left(a, x_{0}\right)$ can be well approximated by

$$
f\left(a, x_{0} \gg 1\right)=x_{0} \frac{\operatorname{ArcSinh}[a]-\log \left[\frac{\sqrt{1+a^{2}}-1}{a}\right]}{\sqrt{1+a^{2}}}-\frac{\log \left[2 x_{0}\right]}{a}
$$

indicating that, for $x_{0} \gg 1, \Delta L$ scales like $z_{0} \beta$, that is

$$
\begin{aligned}
\Delta L= & \frac{4 Q c}{I_{a}} \frac{z_{0} \beta}{R^{*} L^{*} \gamma^{4}} \\
& \times\left\{\frac{\operatorname{ArcSinh}[a]-\log \left[\frac{\sqrt{1+a^{2}}-1}{a}\right]}{\sqrt{1+a^{2}}}-\frac{\log \left[2 \mathrm{x}_{0}\right]}{\mathrm{x}_{0} \mathrm{a}}\right\} .
\end{aligned}
$$

It is interesting to notice that for $x_{0} \ll 1$, namely, $R \approx R^{*}$, $\Delta L$ becomes

$$
\Delta L=\frac{2 Q c z^{2}}{I_{a} \gamma^{4} R^{*} L^{*}}
$$

showing that (6) is a generalization of a previous result [7] derived by assuming $R=R^{*}$ and $L=L^{*}$ (i.e., a parallel envelope at constant bunch length).

Taking for example a $Q=16 \mathrm{pC}$ bunch at $\gamma=20(T=$ $9.7 \mathrm{MeV}$ ), focused down to a $R^{*}=50 \mu \mathrm{m}$ spot and length $L^{*}=30 \mu \mathrm{m}$, starting from a lens located $z^{\prime}=15 \mathrm{~cm}$ from the focal point, with a length $L_{0}=150 \mu \mathrm{m}$, and $\beta=5 \mathrm{~mm}$, one finds the perturbative bunch lengthening $\Delta L$ predicted by (6) (with $x_{0}=30, a=0.13$ ) to be $\Delta L=7 \mu \mathrm{m}$.

We have neglected the possibility of the laser fields acting directly on the beam electrons (i.e., in vacuum). In the case of a short driving laser pulse, the electrons would trail and not overlap with the laser, so there would be no interaction. If a long laser pulse is used as in near term $\mathrm{CO}_{2}$-laser experiments, however, then the laser may interact with the beam electrons. The pondermotive force of the laser tends to bunch the electrons longitudinally into the nulls of the laser beats [9] and to scatter the electrons radially [10].

\section{ACKNOWLEDGMENT}

Thanks to T. Peters for assistance with figures and S. Mistry for manuscript preparation.

\section{REFERENCES}

[1] P. Kung et al., "Generation and measurement of 50-fs (rms) electron pulses," Phys. Rev Lett., vol. 73, pp. 967-970, 1994.

[2] B. E. Carlsten and S. J. Russell, submitted to Phys. Rev. E.

[3] E. Esarey et al., "Overview of plasma-based accellerator concepts," this issue, pp. 252-288.

[4] C. E. Clayton and L. Serafini, "Generation and transport of ultrashort phase-locked electron bunches to a plasma beat wave accelerator," this issue, pp. 400-408. 
[5] R. Ruth et al., Particle Accel., vol. 17, p. 171, 1985.

[6] J. Rosenzweig, N. Barov, and E. Colby, "Pulse compression in RF photoinjectors: Applications to advanced accelerators," this issue, pp. $409-420$.

[7] L. Serafini, "Micro-bunch production with RF photo-injectors," this issue, pp. $421-427$.

[8] D. Gordon, private communication.

[9] F. V. Hartemann et al., "Nonlinear pondermotive scattering of relativistic electrons by an intense laser field at focus," Phys. Rev. E, vol. 51(5), pp. 4833-4843, 1995.

Thomas C. Katsouleas (M'88-SM'91-F'96), for a photograph and biography, see this issue, p. 251.

C. E. Clayton, photograph and biography not available at the time of publication.

L. Serafini, for a photograph and biography, see this issue, p. 408 .
C. Pellegrini, photograph and biography not available at the time of publication.

C. Joshi (M'83-SM'88-F'93), photograph and biography not available at the time of publication.

J. Dawson, photograph and biography not available at the time of publication.

P. Castellano, photograph and biography not available at the time of publication. 